\title{
Motor resonance and linguistic focus
}

\author{
Lawrence J. Taylor and Rolf A. Zwaan \\ Florida State University, Tallahassee, FL, USA
}

\begin{abstract}
Previous studies have demonstrated that verbal descriptions of actions activate compatible motor responses (Glenberg \& Kaschak, 2002; Zwaan \& Taylor, 2006). The present study replicates previous findings showing that, within a sentence, such activation is localized on the verb that denotes the action. Moreover, motor resonance is found to yield to linguistic focus. If a postverbal adverb maintains focus on a matching action ("slowly" or "quickly"), motor resonance occurs, but if the adverb shifts the focus to the agent (e.g., "obediently" or "eagerly"), a cessation of motor resonance ensues. These findings are discussed within the context of theories of motor resonance, action understanding, mental simulation, and linguistic focus.
\end{abstract}

Keywords:

Evidence from neuroscience suggests that both the performance of actions and the recognition of the actions of conspecifics produce motor resonance in primates (Keysers \& Perrett, 2004). For example, the motor cortex is active whether a monkey grasps an object, observes an experimenter grasping an object (Gallese, Fadiga, Fogassi, \& Rizzolatti, 1996), watches a hand go behind a screen that occludes an object (Umiltà et al., 2001), or hears a nut being cracked (Kohler et al., 2002). These findings generalize to humans with the important qualification that such instances of motor resonance seem to occur reliably only when the action falls within an individual's action repertoire (Buccino et al., 2004; Calvo-Merino, Glaser, Grezes, Passingham, \& Q2 Haggard, 2005).

When such evidence from neuroscience is considered with theories that propose a strong link between the performance and conceptual understanding of actions (Prinz, 1997; Rizzolatti \& Craighero, 2004; Wilson \& Knoblich, 2005), the involvement of the motor system is expected during the comprehension of language that describes actions (Rizzolatti \& Arbib, 1998). Indeed, previous studies have shown that sentences describing simple motor actions both facilitate compatible motor responses (Glenberg \& Kaschak, 2002) and activate the brain regions that are active when similar actions (i.e., those that involve the same effector) are performed (de Vega, Robertson, Glenberg, Kaschak, \& Rinck, 2004; Tettamanti et al., 2005).

Other results have shown that individual words that denote actions yield similar behavioural (Zwaan \& Taylor, 2006) and neural effects (Hauk, Johnsrude, \& Pulvermüller, 2004; Pulvermüller, Hauk, Nikulin, \& Ilmoniemi, 2005). Along

Correspondence should be addressed to Larry J. Taylor or Rolf A. Zwaan, Department of Psychology, Florida State University, Tallahassee, FL 32306-1270. E-mail: 1taylor@psy.fsu.edu or zwaan@psy.fsu.edu

We thank Christine de Jesus, Quanne Coombs, and Helena Yardley for assistance with data collection. This research was supported by Grants MH-63972 from the National Institutes of Health and BCS-0446637 from the National Science Foundation. 
similar lines, studies in the action literature have shown that the presentation of an irrelevant word (e.g., "large" or "small") subtly influences the dynamics of a goal-directed action (e.g., grip aperture of a participant's hand) while the participant reaches for an object in anticipation of grasping it (Gentilucci \& Gangitano, 1998; Glover \& Dixon, 2002). Similar effects have been found for incidentally presented nouns (e.g., "baseball" or "tweezers") that are either larger or smaller than the target object (Glover, Rosenbaum, Graham, \& Dixon, 2004). Such effects offer support to the claim that the meanings of words and the affordances (Gibson, 1979) of manipulable objects that nouns can denote produce subtle, but immediate, effects in the motor system of a person who comprehends them.

Most importantly, in some experiments these effects have been demonstrated with a relatively high degree of temporal resolution during the processing of action sentences. Compatible responses are facilitated as soon as constraining information becomes available, before an entire sentence (Chambers, Tanenhaus, Eberhard, Filip, \& Carlson, 2002; Zwaan \& Taylor, 2006) has been presented. These findings offer support to the notion that understanding actions through language relies on mental simulation of the described action and that mental simulation of actions is driven by motor resonance.

Most of the work on language-induced motor resonance has examined the effects of single words or entire sentences. In a recent study (Zwaan \& Taylor, 2006) we examined motor resonance as it unfolds during the comprehension of a sentence. Two key findings with respect to the online profile of motor resonance to emerge from this study were that motor resonance (a) occurs immediately (i.e., as soon as enough specificity is provided by the linguistic context up to that point), and (b) motor resonance is short-lived (i.e., it does not extend beyond the action-specifying verb). The first finding is consistent with theories that view language comprehension as an incremental process, in which information is activated immediately, rather than after a particular chunk of linguistic information (e.g., a phrase or a sentence) has been processed (Chambers et al., 2002). The second finding is the focus of the current article.

Why was motor resonance short-lived in Experiments 4 and 5 of the Zwaan and Taylor (2006) paper? It is instructive to reexamine a representative item from those experiments: After/ lighting/the candles/for the/romantic/evening/he/ dimmed/the/lights.

The target word here is dimmed, which produced motor resonance. The next part of the sentence shifts attention away from the action itself to its result or to the patient of the action. We speculated that this shift of attention was responsible for the extinction of motor resonance. This shift hypothesis is consistent with MacWhinney's (2005) perspectival framework, according to which multiple "perspective shifts" occur as a person reads a sentence. These perspective shifts occur between linguistic constituents that code for different elements of the referential situation (e.g., location, objects, and events) that a body of text describes. When these different elements are combined to form a coherent representation, comprehension is successful (MacWhinney, 2005). Here, we postulate the linguistic focus hypothesis (LFH). According to the LFH, motor resonance falls under the scope of linguistic focus. As long as the action is within linguistic focus, motor resonance occurs. However, as soon as the focus shifts, the mental simulation shifts along with it. The LFH makes sense in light of the common assumption of the cognitive system as a satisficer, not engaging in more activity than is minimally required to perform the task.

Combined with previous findings on motor resonance and mental simulation, the LFH makes specific predictions about the localization of facilitated motor processes during language comprehension. Consider the sentence While at the gas station, he selected unleaded and opened the gas tank (Zwaan \& Taylor, 2006, Exp. 4). According to the LFH, motor resonance for anticlockwise manual rotation is limited to the verb "opened" (which describes an act of anticlockwise manual rotation) because the subsequent linguistic content shifts focus away from that particular action to other elements of the referential situation 
(namely, the acted-upon object). If this is a correct explanation, then when the subsequent content continues to focus on the action, as the adverb "slowly" does in the sentence He placed his hand on the gas cap, which he opened slowly, then a continuation of the motor simulation should be observed. Experiment 1 was designed to test this prediction.

\section{EXPERIMENT 1}

Participants were presented with the critical sentences shown in Appendix A. For each experiment, the paradigm used by Zwaan and Taylor (2006, Exp. 4) was used. Participants read sentences by turning a knob continuously during the frame-by-frame presentation of a sentence. Words were presented in groups of one to three. Every 5 degrees of rotation caused a group of centrally presented words to be replaced by the next group of words in the sentence. On critical trials, a sentence describing an act of manual rotation (e.g., The runner/was very/thirsty./A fan/handed $\mathrm{bim} / \mathrm{a}$ bottle/of cold/water/which be/opened/ quickly, with slashes indicating the boundaries between frames) was presented. For each item, the 10th frame presented the critical verb, and the 11th frame presented the adverb intended to keep the action within linguistic focus. Participants read sentences about manual rotation that were either diagnostically clockwise or anticlockwise while turning a knob either clockwise or anticlockwise. If our prediction generated from the LFH is supported, we should find a significant match advantage not only on the verb, but also on the subsequent adverb.

\section{Method}

\section{Participants}

A total of 73 undergraduate psychology students participated in the experiment for course credit. The data for 3 participants were eliminated due to accuracy below $85 \%$ on the comprehension questions $(M=95.6 \%, S D=5.1$, for both experiments) and the data for 2 participants were eliminated because they were not native English speakers. The final analysis included data from 68 participants.

\section{Apparatus and design}

The apparatus, design, and sentences from a previous study (Zwaan \& Taylor, 2006) were adapted for this experiment. Each item described an act of direction-specific manual rotation (see Appendix A). Items were presented in random order. All sentences were constructed so that they consisted of 11 frames. The 10th frame of each sentence contained the verb, and the 11th frame contained the adverb. Each sentence was designed so that the direction of rotation was as unambiguous as possible by the time the verb appeared. Words were presented in black text on a white background, left justified in the centre of the screen.

A knob that allowed rotation-contingent, subject-paced text presentation was used in both experiments (see also Zwaan \& Taylor, 2006). The knob contained springs that returned it to the centred position when released. As the knob was turned from the centre position, the computer logged a keypress response approximately every 5 degrees. Each key press logged a reading time for a given frame of text and resulted in the presentation of the next frame. Manual rotation direction was manipulated within participants. The linguistically implied rotation direction and manual rotation direction were counterbalanced across four lists. There were 17 participants on each list.

\section{Procedure}

Participants read sentences by turning the knob in either direction (clockwise or anticlockwise). For the first half of the experiment, they turned the knob in one direction to proceed through the sentences and then switched direction for the second half. After each sentence, participants released the knob so that it returned to the centre position. Each participant read 48 sentences (16 experimental, 32 filler) during the experiment. A yes-no comprehension question pertaining to the content of the immediately preceding sentence followed half of the filler items. Participants 
responded to these comprehension questions using a standard keyboard.

The experiment began with a participant seated in front of a computer monitor, a keyboard, and a knob wired to the keyboard. After sitting, the participant laid the keyboard across his or her lap to answer comprehension questions. The knob remained on the desk and centred in front of the monitor for the duration of the experiment. Before the experiment began, each participant completed 20 practice trials under experimenter supervision. The experimenter made sure that participants were turning the knob smoothly throughout the duration of each sentence instead of doing the task with repetitive, jerking motions. After the practice trials, every participant was judged to be able to do the task well enough to proceed. Most participants reached this criterion after four or five practice sentences.

A trial began with the knob at the centre position and the first frame of text of a sentence presented on the screen. When the participant turned the knob in the correct direction for approximately 5 degrees, the second frame of text was presented. When the participant turned the knob an additional 5 degrees, the third frame of text was presented. This continued until the 11th frame, at which point the participant either was instructed to release the knob and wait for the next sentence or was presented with a comprehension question. Questions required a response on the keyboard.

\section{Results}

Mean reading times for the critical regions are Q3 shown in Figure 1. Segment reading times $<75 \mathrm{~ms}$ and $>2,000 \mathrm{~ms}$ were removed from the analysis as well as times more than 3 standard deviations from a subject's cell mean. In total less than $1 \%$ of the observations were removed. All analyses used mean reading times. Initial analyses of variance (ANOVAs) with list (a between-participants factor) showed that interactions between this factor and match (when the direction of a participant's manual rotation matched the direction of the manual rotation described by a sentence) all had $p$-values greater than .15 , so this factor was dropped from further analysis, and $t$ tests were used (Pollatsek \& Well, 1995).

Directional tests showed that there was match advantage on the verb by participants, $t_{1}(67)=$ $1.69, p<.05$, and by items, $t_{2}(15)=1.77$, $p<.05$, and, crucial to our hypothesis, also on the adverb, $t_{1}(67)=2.08, p<.025 ; t_{2}(15)=$ 1.57, $p<.07$. There were no significant effects on the preceding segments $(p s>.16)$.

\section{Discussion}

These results support the LFH. In addition to finding motor resonance on the verb describing the action, a finding that replicates Zwaan and Taylor's (2006) Experiment 4, we now also found motor resonance on adverbs that modified the described action and immediately followed the verb.

\section{EXPERIMENT 2}

The adverbs in Experiment 1 primarily modified the described manual rotation. In Experiment 2, those action-modifying adverbs were replaced with agent-modifying adverbs: words that did not primarily modify the action (e.g., happily, eagerly, or nervously). These adverbs denote information that is most relevant to the mental or motivational state of the protagonist performing the action, not the action itself. This manipulation is compatible with linguistic taxonomies of adverbs that draw a distinction between subject-oriented

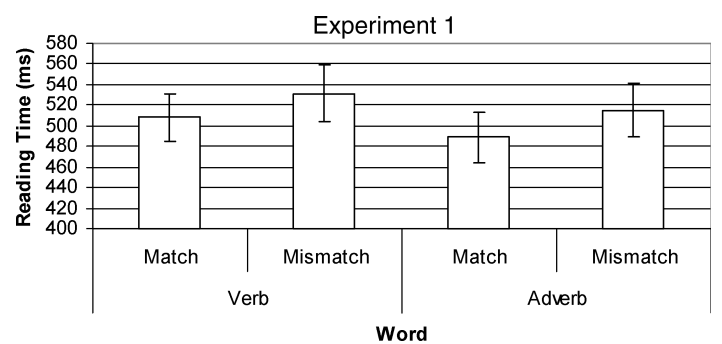

Figure 1. Mean reading times and standard errors for the critical regions in Experiment 1. 
adverbs and process- or manner-oriented adverbs (Jackendoff, 1972; Nakamura, 1997). In Experiment 2, the methods from Experiment 1 were repeated with the exception that we replaced adverbs that primarily modify actions with adverbs that do not (see Appendix B) as discussed above.

\section{Method}

\section{Participants}

A total of 64 undergraduate psychology students participated in the experiment for course credit. The data for 1 participant were eliminated due to accuracy below $85 \%$ on the comprehension questions, and the data for 3 participants were eliminated because they had cell means that were greater than 3 standard deviations from the mean reading times for all participants. The final analysis included data from 60 participants.

\section{Apparatus and design}

The apparatus and design from Experiment 1 were used in Experiment 2, with the exception that the adverbs were replaced (see Appendix B).

\section{Procedure}

The procedure from Experiment 1 was repeated.

\section{Results}

Mean reading times for the critical regions are Q4 shown in Figure 2. As in Experiment 1, segment reading times $<75 \mathrm{~ms}$ and $>2,000 \mathrm{~ms}$ were removed from the analysis as well as times more

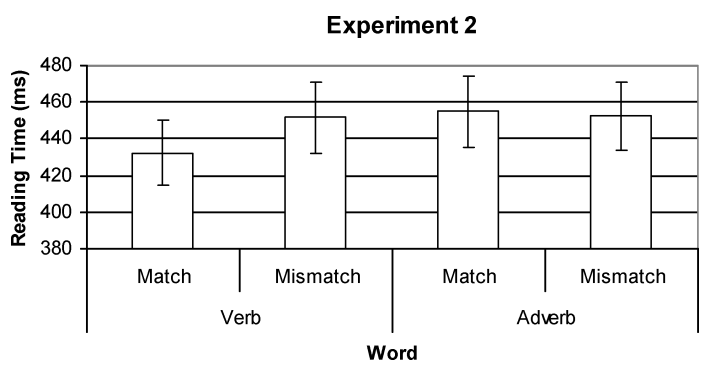

Figure 2. Mean reading times and standard errors for the critical regions in Experiment 2. than 3 standard deviations from a subject's cell mean. In total $1.25 \%$ of the observations were removed.

Initial ANOVAs with list showed that interactions between this factor and match all had $p$-values greater than .15 , so this factor was dropped from further analysis, and $t$ tests were used (Pollatsek \& Well, 1995).

Directional tests showed that there was a match advantage on the verb by participants and a marginally significant match advantage by items, $t_{1}(59)=2.59, p<.025 ; t_{2}(15)=1.87, p=.08$, but not on the adverb, $t_{1}(59)=0.824, p=.41$; $t_{2}(15)=0.786, p=.44$. There were no significant effects on the preceding segments $(p s>.24)$. The lack of a match effect on the adverb was not due to a lack of statistical power. The power to detect a 25-ms match advantage, as observed in Experiment 1, was .91 for a one-tailed test (Lenth, 2006).

\section{Discussion}

These results replicate the match advantage on the verb observed in Experiment 1, but show that the match advantage we found on action-modifying adverbs did not occur if the adverb does not primarily modify the action that is described before it.

\section{GENERAL DISCUSSION}

The results from these two experiments support predictions made by the LFH. When a verb is modified by an adverb, compatible motor responses are facilitated on the adverb only if it primarily modifies the action (e.g., quickly and slowly) and not when some other element of the referential situation is modified (e.g., happily, eagerly, or nervously). Experiment 1 represents an initial attempt to extend the localized motor resonance effect (Zwaan \& Taylor, 2006) from the verb to an adverb that immediately follows it. Compatible responses were faster on the verb as well as on the subsequent action-modifying adverb. This was not the case with Experiment 2, 
in which the action-modifying adverbs were replaced with agent-modifying adverbs.

The primary contribution of this article is confirmation of a prediction made by a synthesis of the $\mathrm{LFH}$ and previous findings on the localization of motor resonance during language processing (Zwaan \& Taylor, 2006). If the previous finding that motor resonance is localized on action verbs is due to the surrounding content shifting focus away from the action, then maintaining focus on the action by following the verb with an actionmodifying adverb should cause motor resonance to extend beyond the verb to the adverb. Our experiments support this prediction.

Results discussed earlier (Gentilucci \& Gangitano, 1998; Glenberg \& Kaschak, 2002; Glover et al., 2004; Zwaan \& Taylor, 2006, Exp. 2) suggest that language affects motor processes in a top-down fashion in that the higher order process of understanding words or the actions described by sentences affects subsequent motor activity. However, a bottom-up effect is not only plausible, but consistent with previous findings (Lindemann, Stenneken, van Schie, \& Bekkering, 2006; Zwaan \& Taylor, 2006, Exp. 4) and the present experiments, in which participants perform an action in order to indicate that they have read a consistent or inconsistent word. For example, previous studies have shown that when participants form an intention to act (e.g., to pick up a magnifying glass and move it towards one's eye) before the presentation of a semantically related word ("eye"), they are faster to respond to the word in a task that invites semantic processing of the word, such as categorization or lexical decision, but not when the task does not invite semantic processing, such as letter detection (Lindemann et al., 2006). As with the current study, while bottom-up processing is consistent with the results, top-down processes could still explain the findings, as the word response is confounded with the compatible action. While this is an issue that warrants further investigation, either top-down processes or bottom-up processes or both would be consistent with a claim that the semantic and motor systems rely on partially overlapping neurophysiological substrates. For example, results showing that visually perceived rotation affects manual rotation (Zwaan \& Taylor, 2006, Exp. 1) coupled with previous findings that manual rotation affects perception of an ambiguously rotating visual stimulus suggest that manual rotation and perception of visual rotation share common neural systems (Wohlschläger, 2000).

Several alternative explanations for our results could be proposed, but are demonstrably unviable. Possible alternative explanations for the results include: (a) They are due to demand effects (participants were somehow aware of the manipulation, and this drove the differences of interest); (b) the effect on the adverb is merely a continuation of the original effect on the verb and does not reflect the influence of the adverb on maintaining focus on the action; (c) there was a confound between the items that actually caused the differences of interest; and (d) the effect on the adverb is really a sentence wrap-up effect. Each alternative explanation is considered and addressed in turn.

First, a sceptic could argue that participants became aware of the intention behind the experiment since they were engaging in manual rotation while reading sentences about manual rotation. To prevent this from becoming an issue, the critical items were embedded inside a larger set of similarly worded items describing similarly mundane actions. When probed during postexperiment interviews, no participant reported having any knowledge of the manipulation. Further, even if correct, this would be an especially odd alternative explanation for the differences found on the adverb. A substantial proportion of the participants would have had to have been sensitive to the distinction between action- and agent-modifying adverbs in order for this to explain the pattern found in the data.

Second, a sceptic could dismiss the findings on the adverb as merely a continuation of the original effect. According to this criticism, any word appearing directly around the verb is subject to "spillover" motor resonance effects. This is an important criticism to counter, since the claim made here is that the result on the adverb supports the LFH. If it were the case that the verb simply influenced surrounding words regardless of their 
content, then there would be an effect on the agent-modifying adverbs in Experiment 2 or on the direct object that directly followed the verb in previously reported experiments (Zwaan \& Taylor, 2006, Exps. 4 and 5).

Third, it might be argued that the use of adverbs such as quickly and slowly should yield different response times given that they explicitly describe the speed with which the described action is performed. Although this is an interesting idea that is perhaps worth pursuing in a more sensitive paradigm, it is irrelevant to the current results, since any differences between items, other than the intended differences between conditions, were negated through counterbalancing. In other words, those differences are orthogonal to the manipulation and differences of interest.

Fourth, one could argue that, because the adverb was the last word in the sentence, the effect on that word is attributable to a motor resonance effect for the entire sentence (as in Experiments 2 and 3 in Zwaan \& Taylor, 2006, or in Glenberg \& Kaschak, 2002) and not continued focus on the action. However, if the last word of a sentence showed such an effect regardless of its content, then that pattern would have been found on the last word of other experiments in which the last word was not an action-modifying adverb (Experiment 2 in this paper and Experiments 4 and 5 from Zwaan \& Taylor, 2006).

Although beyond the scope of the present article, these results invite future investigations into the specificity of language-induced motor resonance and the importance of motor resonance in providing the underpinnings of action understanding. For example, the distinction between fast and slow action modifiers (e.g., quickly vs. slowly) could be one that produces detectable differences in motor resonance. Additionally, adverbs that disambiguate the direction (e.g., upwards in the sentence He moved his hand upwards) of an action could show independent localization of motor resonance (that is, facilitation for compatible responses on the adverb only, not the verb). A related paradigm involves changing the position of the adverb. In the present experiments, the adverb directly follows the verb. An experimenter might predict no effect at all when the adverb precedes the direction-disambiguating verb (... he quickly screwed in the light bulb) but a reemergence of motor resonance when a direct object interrupts the focus on the action (.. he screwed in the light bulb quickly). Future research may address these issues.

The present results show that a verbal description of an action leads to a very subtle pattern of motor activation in the comprehender, which has not been shown previously. An important qualification of this research and other studies showing motor resonance during or after language comprehension is that this does not constitute direct evidence for the claim that action comprehension relies on a mental simulation (Rizzolatti \& Craighero, 2004; see also the review article in this issue) of that action, though the present results are compatible with such a claim. Evidence for mental simulation requires showing that an action described by a sentence is facilitated by reading it. The present, and similar, results show a difference between matching and mismatching conditions, a result that is compatible with either facilitation of the matching condition or interference of the mismatching condition. We are currently running studies, which compare neutral, matching, and mismatching actions, that will shed light on this issue.

First published online day month year

\section{REFERENCES}

Buccino, G., Lui, F., Canessa, N., Patteri, I., Lagravinese, G., Benuzzi, F., et al. (2004). Neural circuits involved in the recognition of actions performed by nonconspecifics: An fMRI study. Journal of Cognitive Neuroscience, 16, 114-126.

Calvo-Merino, B., Glaser, D. E., Grezes, J., Passingham, P. E., \& Haggard, P. (2005). Action observation and acquired motor skills: An fMRI study with expert dancers. Cerebral Cortex, 15, 1243-1249.

Chambers, C. G., Tanenhaus, M. K., Eberhard, K. M., Filip, H., \& Carlson, G. N. (2002). Circumscribing referential domains in real-time sentence comprehension. Journal of Memory and Language, 47, 30-49. 
de Vega, M., Robertson, D. A., Glenberg, A. M., Kaschak, M. P., \& Rinck, M. (2004). On doing two things at once: Temporal constraints on actions in language comprehension. Memory $\mathcal{E}^{\circ}$ Cognition, 32, 1033-1043.

Gallese, V., Fadiga, L., Fogassi, L., \& Rizzolatti, G. (1996). Action recognition in the premotor cortex. Brain, 119, 593-596.

Gentilucci, M., \& Gangitano, M. (1998). Influence of automatic word reading on motor control. European Journal of Neuroscience, 10, 752-756.

Gibson, J. J. (1979). The ecological approach to visual perception. Boston, MA: Houghton-Mifflin.

Glenberg, A. M., \& Kaschak, M. P. (2002). Grounding language in action. Psychonomic Bulletin \& Review, 9, 558-565.

Glover, S., \& Dixon, P. (2002). Semantics affect the planning but not control of grasping. Experimental Brain Research, 146, 383-387.

Glover, S., Rosenbaum, D. A., Graham, J., \& Dixon, P. (2004). Grasping the meaning of words. Experimental Brain Research, 154, 103-108.

Hauk, O., Johnsrude, I., \& Pulvermüller, F. (2004). Somatotopic representation of action words in human motor and premotor cortex. Neuron, 41, 301-307.

Jackendoff, R. (1972). Semantic interpretation in generative grammar. Cambridge, MA: MIT Press.

Keysers, C., \& Perrett, D. I. (2004). Demystifying social cognition: A Hebbian perspective. Trends in Cognitive Sciences, 8, 501-507.

Kohler, E., Keysers, C., Umiltà, M. A., Fogassi, L., Gallese, V., \& Rizzolatti, G. (2002). Hearing sounds, understanding actions: Action representation in mirror neurons. Science, 297, 846-848.

Lenth, R. V. (2006). Java Applets for Power and Sample Size [Computer software]. Retrieved December 13, 2006, from http://www.stat.uiowa.edu/ rlenth/ Power

Lindemann, O., Stenneken, P., van Schie, H. T., \& Bekkering, H. (2006). Semantic activation in action planning. Journal of Experimental Psychology: Human Perception and Performance, 32, 633-643.

MacWhinney, B. (2005). The emergence of grammar from perspective taking. In D. Pecher \& R. A. Zwaan (Eds.), Grounding cognition: The role of perception and action in memory, language, and thinking (pp. 198-223). Cambridge, UK: Cambridge University Press.

Nakamura, W. (1997). A cognitive approach to English adverbs. Linguistics, 35, 247-287.
Pollatsek, A., \& Well, A. D. (1995). On the use of counterbalanced designs in cognitive research: A suggestion for a better and more powerful analysis. Journal of Experimental Psychology: Learning, Memory, \& Cognition, 21, 785-794.

Prinz, W. (1997). Perception and action planning. European Journal of Cognitive Psychology, 9, 129-154.

Pulvermüller, F., Hauk, O., Nikulin, V. V., \& Ilmoniemi, R. J. (2005). Functional links between motor and language systems. European Journal of Neuroscience, 21, 793-797.

Rizzolatti, G., \& Arbib, M. A. (1998). Language within our grasp. Trends in Neurosciences, 21, 188-194.

Rizzolatti, G., \& Craighero, L. (2004). The mirrorneuron system. Annual Review of Neuroscience, 27, 169-172.

Tettamanti, M., Buccino, G., Saccuman, M. C., Gallese, V., Danna, M., Scifo, P., et al. (2005). Listening to action-related sentences activates frontoparietal motor circuits. Journal of Cognitive Neuroscience, 17, 273-281.

Umiltà, M. A., Kohler, E., Gallese, V., Fogassi, L., Fadiga, L., Keysers, C., et al. (2001). "I know what you are doing": A neurophysiological study. Neuron, 32, 91-101.

Wilson, M., \& Knoblich, G. (2005). The case for motor involvement in perceiving conspecifics. Psychological Bulletin, 131, 460-473.

Wohlschläger, A. (2000). Visual motion priming by invisible actions. Vision Research, 40, 925-930.

Zwaan, R. A., \& Taylor, L. (2006). Seeing, acting, understanding: Motor resonance in language comprehension. Journal of Experimental Psychology: General, 135, 1-11.

\section{APPENDIX A}

\section{Critical sentences for Experiment 1}

\section{Clockwise}

He had/been on/the highway/for a/long time./ When he/saw a gas/station,/he/exited/slowly

During the/film,/the light/bulb/burned out./ He found/a new /light bulb/which he/screwed in/rapidly

The gardener/noticed/that the/water/was still/running/He approached/the faucet/which he/turned off/quickly 
The good/student/was about/to take/the $\mathrm{SAT}$./He/picked up/his pencil/which he/sharpened/rapidly

The man/was/replacing/his tire./He placed/ onto/the tire/a lugnut/which he/tightened/ slowly

He hopped/into his car,/very late/for work./ He placed/the key/into/the ignition/which he/ started/quickly

He was/about to/attach the last/leg onto the/ table./He picked up/the/screwdriver/and/ screwed in/slowly

He wanted/to read/from his/favorite/book./ He sat/next to/a lamp/which he/turned on/ quickly

\section{Anticlockwise}

He was/craving a /juicy/pickle./On the/shelf, he/found a/closed jar/which he/opened/rapidly

$\mathrm{He}$ selected/unleaded/at the/gas station./He placed/his hand/on the /cap/which he/opened/ slowly

His father/walked /into/the room./He/ noticed/the loud/volume/which he/turned down/gradually

$\mathrm{He}$ wanted/to try/his new/satellite TV./ Behind the/TV, he/grabbed the/cable/which he/unscrewed/quickly

The runner/was very/thirsty./A fan/handed him/a bottle/of cold/water/which he/opened/ quickly

He waited/at the /intersection/before he/could turn./He saw/an/opening/and/turned left/slowly

The chicken/in the oven/looked cooked/perfectly./The cook/walked/over to/the oven/ which he/turned down/slowly

He lit/the candles/for the/romantic/evening./ He noticed/the bright/lights/which he/dimmed/ slowly

\section{APPENDIX B}

\section{Critical sentences for Experiment 2}

\section{Clockwise}

He had/been on/the highway/for a/long time./ When he/saw a gas/station, /he/exited/eagerly
During the/film,/the light/bulb/burned out./ $\mathrm{He}$ found/a new /light bulb/which he/screwed in/carefully

The gardener/noticed/that the/water/was still/running/He approached/the faucet/which he/turned off/thoughtfully

The good/student/was about/to take/the $\mathrm{SAT}$./He/picked up/his pencil/which he/sharpened/nervously

The man/was/replacing/his tire./He placed/ onto/the tire/a lugnut/which he/tightened/ skillfully

He hopped/into his car,/very late/for work./ He placed/the key/into/the ignition/which he/ started/hastily

$\mathrm{He}$ was/about to/attach the/last leg/onto/the table./He picked up/a screw/which he/screwed in/patiently

He wanted/to read/from his/favorite/book./ He sat/next to/a lamp/which he/turned on/ eagerly

\section{Anticlockwise}

He was/craving a/juicy/pickle./On the/shelf, he/found a/closed jar/which he/opened/hungrily

$\mathrm{He}$ selected/unleaded/at the/gas station./He placed/his hand/on the /cap/which he/opened/ carefully

His father/complained/about/the noise./ John/walked up/to the/stereo/which he/turned down/obediently

$\mathrm{He}$ wanted/to try/his new/satellite TV./ Behind the/TV, he/grabbed the/cable/which he/unscrewed/hastily

The runner/was very/thirsty./A fan/handed him/a bottle/of cold/water/which he/opened/ eagerly

$\mathrm{He}$ waited/at the /intersection/before he/ could turn./He saw/an/opening/and/turned left/skillfully

The chicken/in the oven/looked cooked/to perfection./The cook/walked/over to/the oven/ which he/turned down/happily

He lit/the candles/for the/romantic/evening./ He noticed/the bright/lights/which he/dimmed/ carefully 


\section{PQJE262401}

Queries

Lawrence J. Taylor and Rolf A. Zwaan

Q1 Please supply up to 5 keywords.

Q2 Calvo-Merino et al., 2004, ok as changed to Calvo-Merino, Glaser, Grezes, Passingham, \& Haggard, 2005?

Q3 No text reference to Figure 1: ok as inserted here?

Q4 No text reference to Figure 2: ok as inserted here?

Q5 Please give specific citation, and list in references. Is it Fischer? 